\title{
Non-Drifting Limb Angle Measurement Relative to the Gravitational Vector During Dynamic Motions Using Accelerometers and Rate Gyros
}

\author{
Andrew J. Petruska and Sanford G. Meek
}

\begin{abstract}
A method for estimating limb orientation, during static, quasi-static, and dynamic motions, by using a combination of gyroscopes and accelerometers is presented. The method uses two tri-axis accelerometers and one single axis rate gyro to calculate an estimate of angle relative to the gravitational vector independently from the rotational accelerations. This unbiased inclination estimate is blended with the angular velocity and acceleration measurements using a Kalman filter to obtain the final estimated orientation. Initially developed for implementation in a feedback control loop for control of sit-tostand transitions in felines during direct electrical stimulation of the sciatic nerve, the method can be directly applied to other limb angle measurement tasks such as human gait analysis. The algorithm and sensor is tested on a rotational linkage with a predefined trajectory and compared to an encoder measurement with good agreement.
\end{abstract}

\section{INTRODUCTION}

Measuring joint angles during dynamic motions is required for feedback control of motion in both robotic manipulators and neuro-muscular control subjects. In robotic applications, joint angles are most directly obtained from the use of encoders or other electro-mechanical devices built into the joint. In non-robotic structures, such as a feline hind limb, joint angle measurement is more difficult and direct access to the joint for measurement is often not available. Research into joint angle measurement in the past has focused primarily on human gait analysis to aid with many things from development of new prostheses to rehabilitation of spinal cord injury and stroke patients. The techniques for measuring joint angles developed for gait analysis are directly applicable for motion control so long as they can be implemented in real time. Currently, most gait measurements take place in a controlled laboratory environment using optical systems or goniometers. Researchers have been investigating ways to instrument patients for gait analysis in non-laboratory conditions; some of the the pioneering efforts to integrate MEMS sensors into gait analysis were performed by [1] and [2].

The MEMS systems use a combination of rate gyros and accelerometers to estimate the angle of each limb during a gait cycle. The concept is as follows: Measurements of the angular velocity obtained from a rate gyro are integrated over time to obtain an angle estimate. Measurements of the gravitational-coupled acceleration are compared to extract the inclination. The two estimates are combined using

This work was supported by the National Science Foundation under grant DGE-0654414.

The authors are with the Department of Mechanical Engineering, University of Utah, Salt Lake City, 84112 Andrew.Petruska@utah.edu, meek@mech.utah.edu various methods to obtain a final estimate of angle. Apart from random noise, the two estimates contain biases arisings from different sources. Integration of the angular velocity results in estimate drift due to small errors in the measured velocities. Estimation of inclination does not suffer from drift; but, it is suspectable to gravitational direction aliasing by the angular and centripetal accelerations on the sensor. In the end, any algorithm is left combining a direct estimate of angle known to be biased during dynamic motions and an integrated estimate of angle known to drift over time. Reconciling these two major limitations in the inclination estimate is the differentiator between the approaches taken to date. There are three basic approaches to mitigating these error modes:

1) Attempt to mitigate drift while ignoring the inclination measurement ([2], [3]).

2) Combine the measurements relying on the integration over short dynamic periods and the and inclination over long static periods ([1], [4], [5], [6], [7], [8], [9]).

3) Removing the angular and centripetal bias by adding additional geometric or previous state information to the system ([10], [11], [12], [13], [14], [15], [16]).

Many of these approaches use Kalman filtering to estimate the angle despite the known biases in the measurement. This filtering methodology is shown in [9] to be less effective than other strategies for dealing with the direct measurement bias; so, implementations relying on combining measurements should address the bias in the inclination measurement or use alternative methods for combining the information.

The above techniques have demonstrated reasonable performance for measuring human gait under walking conditions. The direct application of these techniques for control feedback during sit-to-stand motions in felines, the motivation behind this research, is problematic because of the combination of static, dynamic, and non-cyclic motions. The need to have broad frequency content to capture both the static, dynamic, and transition motions prevents implementation of inclination based techniques discussed above in [1]. Another major limitation in extracting an angle estimate during motion for felines is the rotation axis of the joint moves spatially as the feline transitions from sitting to standing [17]. The sensor system required to fill the needs of the control system has to operate accurately and without drift under both static and dynamic motions and needs to be small enough to be attached to the feline's hind limb and cannot rely on precise mounting information.

To accomplish the stated task a MEMS inertial motion 
unit (IMU) is used similar to the ones investigated above. Two tri-axis accelerometers and one rate gyro are used to measure the angular acceleration and velocity as well as extract inclination relative to the gravitational vector. These three estimates are then fused together by using a Kalman filter. The difference between the technique discussed in this paper and the ones explored previously is the estimation of inclination during dynamic motions presented is not aliased by angular and centripetal acceleration, does not require precise mounting information, and does not require integration. This leads to a filter which can handle both static and dynamic motions without drift or bias.

The rest of the paper will be structured as follows. Section II will review the sensor kinematics and dynamic inclination measurement. Section III outlines the Kalman filter implementation. Section IV discusses the testing performed and the performance of the system. Section V outlines the conclusions and future work to be performed.

\section{UNBIASED DYNAMIC INCLINATION MEASUREMENT}

The IMU used in this method consists of two tri-axial accelerometers and one uni-axial rate gyro. The basic geometry of the setup is shown in Fig. (1). The IMU is intended to be mounted to the rotating limb some distance $L$ from the axis of rotation, which is not known. The distance between the accelerometers is $d L$ and is precisely controlled during manufacturing. The direction along the limb is defined as $x$; the direction tangent to the arc of rotation is defined as $y$; and, the direction perpendicular to $x$ and $y$ is defined as $z$. Without loss of generality, the gravitational vector is constrained to the $x, y$ plane for this discussion. The accelerometers are oriented so they record the accelerations in the $x, y$, and $z$ directions. The measured acceleration will be defined by the accelerometer number first and the axis of measurement second; for example, the acceleration measured by accelerometer number one in the $x$ direction will be denoted as $a_{1, x}$. If the accelerometers are mounted askew from the correct orientation, it is assumed the measured readings will be rotated into the proper frame before implementing the algorithm discussed below. The rate gyro, $R G$, is oriented so it measures angular velocity about the $z$ axis; exact placement of the rate gyro along the IMU is not required as its measurement is independent of placement.

Animal joint kinematics allow the assumption that all joint rotation axes are approximately parallel limiting the accelerations to centripetal, angular, gravitational, and translational (no coriolis terms). It is assumed that the measurements will be taken in an inertial frame; so, any pure translational accelerations have to arise during a gait cycle. Since animal joints are rotary in nature, any purely translational accelerations occur when two or more joints move at exactly the correct rate to cause the linking segment to experience a purely linear motion. It is assumed that this exact rotational rate match-up will occur only momentarily during a gait cycle and can be neglected when calculating the inclination angle. At the moments in the gait cycle where this assumption does not hold, the calculated inclination will be biased as it will report the direction of the vector created by combining the linear acceleration and gravity and not gravity alone. Because a Kalman filter is being used to combine this estimate of inclination with a integration estimate, brief changes in the calculated inclination due to translation acceleration or erroneous sensor measurements will be filtered out. Given these assumptions, the accelerations recorded by the accelerometers are described by (1).

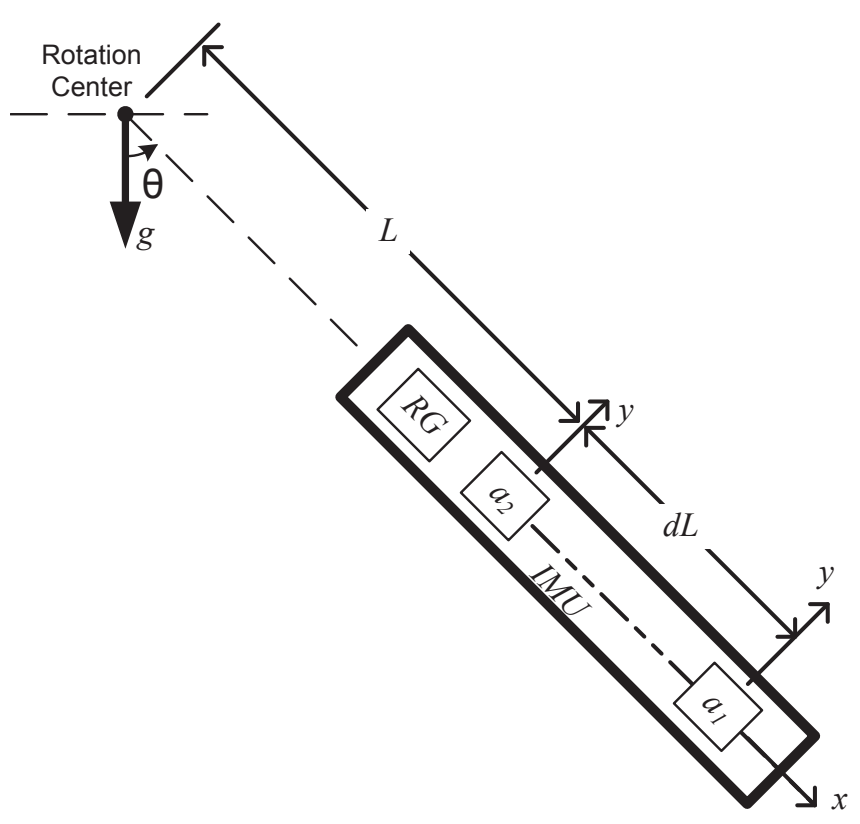

Fig. 1. Generic layout of the IMU Design

$$
\left.\begin{array}{l}
a_{1, x}=(L+d L) \dot{\theta}^{2}+g \cos (\theta) \\
a_{1, y}=-(L+d L) \ddot{\theta}-g \sin (\theta) \\
a_{2, x}=L \dot{\theta}^{2}+g \cos (\theta) \\
a_{2, y}=-L \ddot{\theta}-g \sin (\theta)
\end{array}\right\}
$$

Where $\mathrm{L}$ is defined as the distance from the measurement center of $a_{2}$ to the instantaneous center of rotation as projected onto the $x$ axis; $d L$ is the distance between the measurement centers of $a_{1}$ and $a_{2}$; and, $\theta, \dot{\theta}$, and $\ddot{\theta}$ are, respectively, the angle relative to gravity, the first time derivative of that angle, and the second time derivative of that angle. The difference in the two accelerometer measurements gives the angular acceleration as defined in (2).

$$
\ddot{\theta}=\frac{a_{2, y}-a_{1, y}}{d L}
$$

The relationships defined in (1) can be solved for $\theta$ independent of distance and dynamics in four different solution regimes:
1) Static:
$\dot{\theta}=\ddot{\theta}=0$
2) Constant Velocity:
$\dot{\theta} \neq 0, \ddot{\theta}=0$
3) Initial Acceleration:
$\dot{\theta}=0, \ddot{\theta} \neq 0$
4) Dynamic:
$\dot{\theta} \neq 0, \ddot{\theta} \neq 0$ 
The solution to the static regime, denoted $\theta_{\text {Static }}$, is the trivial solution given in (3) and is the classic method used to measure inclination in the works discussed in the introduction.

$$
\theta_{\text {Static }}=\operatorname{Atan} 2\left(-a_{y}, a_{x}\right)
$$

It can be shown that the solution to both the constant velocity and the initial acceleration regimes is equivalent to the solution to the dynamic regime. The solution to the dynamic regime, denoted $\theta_{\text {Dynamic }}$, is derived as follows:

Rearranging the equations in (1) and solving for length terms gives:

$$
\left.\begin{array}{rl}
L+d L & =\frac{a_{1, x}-g \cos (\theta)}{\dot{\theta}^{2}} \\
L+d L & =-\frac{a_{1, y}+g \sin (\theta)}{\ddot{\theta}} \\
L & =\frac{a_{2, x}-g \cos (\theta)}{\dot{\theta}^{2}} \\
L & =-\frac{a_{2, y}+g \sin (\theta)}{\ddot{\theta}}
\end{array}\right\}
$$

which can be combined to remove the dependence on distance:

$$
\left.\begin{array}{rl}
-\frac{a_{1, y}+g \sin (\theta)}{\ddot{\theta}} & =\frac{a_{1, x}-g \cos (\theta)}{\dot{\theta}^{2}} \\
-\frac{a_{2, y}+g \sin (\theta)}{\ddot{\theta}} & =\frac{a_{2, x}-g \cos (\theta)}{\dot{\theta}^{2}}
\end{array}\right\}
$$

the ratio of these equations removes the dependence on angular acceleration and velocity:

$$
\frac{a_{1, y}+g \sin (\theta)}{a_{2, y}+g \sin (\theta)}=\frac{a_{1, x}-g \cos (\theta)}{a_{2, x}-g \cos (\theta)}
$$

cross multiplying gives:

$$
\begin{array}{r}
a_{1, y} a_{2, x}+a_{2, x} g \sin \theta-a_{1, y} g \cos \theta= \\
a_{2, y} a_{1, x}+a_{1, x} g \sin \theta-a_{2, y} g \cos \theta
\end{array}
$$

which can be rewritten as:

$$
A \cos (\theta)+B \sin (\theta)=D
$$

where:

$$
\begin{aligned}
& A=a_{2, y}-a_{1, y}, \\
& B=a_{2, x}-a_{1, x} \\
& D=\frac{a_{1, x} a_{2, y}-a_{1, y} a_{2, x}}{g}
\end{aligned}
$$

the cosine and sine terms can be combined and can be rewritten as:

$$
A \cos (\theta)+B \sin (\theta)=C \sin (\theta+\delta)=D
$$

where:

$$
\begin{aligned}
& \delta=\operatorname{Atan} 2(A, B), \\
& C=\operatorname{sign}\left(\frac{A}{\sin \delta}\right) \sqrt{A^{2}+B^{2}}, \\
& \operatorname{sign}(x) \equiv \frac{x}{|x|}
\end{aligned}
$$

Finally, (9) can be solved for $\theta_{\text {Dynamic }}$ according to (10).

$$
\theta_{\text {Dynamic }}=\operatorname{Atan} 2\left(D / C, \zeta \sqrt{1-\frac{D^{2}}{C^{2}}}\right)-\delta
$$

where:

$$
\begin{aligned}
& \zeta=\operatorname{sign}[\cos (\hat{\theta}+\delta)] \\
& \hat{\theta}=\text { estimate of current inclination angle. }
\end{aligned}
$$

This calculation of $\theta_{\text {Dynamic }}$ provides an unbiased dynamic measurement of angle relative to the gravitational vector because it is independent of angular and centripetal acceleration and their respective moment arms. Determining when to use $\theta_{\text {Dynamic }}$ or $\theta_{\text {Static }}$ and calculating a $\operatorname{good} \hat{\theta}$ will be discussed in further detail in section III.

\section{KALMAN FILTER IMPLEMENTATION}

The Kalman filter is a statistical filter used to combine estimates of a state in the presence of process, measurement, and disturbance noise. The filter derivation can be found in [18]. The basic premise is as follows. Each system description, a set of states at a given time, is treated as a normal probability distribution with a known estimate $\left(E\left[X_{i-1}\right]\right)$ and Variance $\left(V\left[X_{i-1}\right]\right)$. This estimate and variance are evolved using the state transition matrix to obtain an a priori probability distribution $\left(X_{i-1^{+}}\right)$which is then combined with the measurement probability distribution $\left(X_{m}\right)$ to obtain a final probability distribution $\left(X_{i}=X_{i-1^{+}}+X_{m}\right)$. The expected value of this final probability distribution is the new definition of state and the variance of this distribution is the uncertainty in that estimate. Given the correct definitions for parameter variances and the state transition matrices, this estimate of state is statistically the best possible given all of the previous measurements.

The algorithm provided in [19] for implementation is as follows. Convert the system to be filtered into a set of state space equations as described in (11). $\vec{x}$ is the state vector; $\mathbf{F}$ is the discrete time state transition matrix; $\vec{u}$ is a known control input; $\mathbf{G}$ is the state transition matrix for a control input; $\overrightarrow{1}$ is a column vector of all ones; $\mathbf{V}$ is the process noise matrix; $\mathbf{H}$ is the state visibility matrix; $\mathbf{W}$ is the measurement noise matrix; and, $\vec{y}$ is the system output vector. Given these equations and a set of measurements $\left(\vec{x}_{m}\right)$, a previous state $(\vec{x})$, and a previous state uncertainty matrix $\left(\mathbf{P}_{i}\right)$, the new state estimate $\left(\vec{x}_{i+1}\right)$ and new state uncertainty matrix $\left(\mathbf{P}_{i+1}\right)$ are given in (12).

$$
\left.\begin{array}{rl}
\vec{x}_{i+1} & =\mathbf{F} \vec{x}+\mathbf{G} \vec{u}+\mathbf{V} \overrightarrow{1} \\
\vec{y} & =\mathbf{H} \vec{x}+\mathbf{W} \overrightarrow{1} \\
\vec{x}_{i}^{+} & =\mathbf{F} \vec{x}_{i}+\mathbf{G} \vec{u} \\
\mathbf{P}_{i}^{+} & =\mathbf{F} \mathbf{P}_{i} \mathbf{F}^{T}+\mathbf{V} \\
\vec{x}_{i+1} & =\vec{x}_{i}^{+}+\mathbf{R}\left(\vec{x}_{m}-\mathbf{H} \vec{x}_{i}^{+}\right) \\
\mathbf{P}_{i+1} & =\mathbf{P}_{i}^{+}-\mathbf{R} \mathbf{H} \mathbf{P}_{i}^{+} \\
\text {where: } & \\
\mathbf{S} & =\mathbf{H P}_{i}^{+} \mathbf{H}^{T}+\mathbf{W} \\
\mathbf{R} & =\mathbf{P}_{i}^{+} \mathbf{H S}^{-1}
\end{array}\right\}
$$




\section{A. Parameter Definition}

Two separate Kalman filters are implemented because of the non-linear nature of the angle estimate. The accelerometer measurements are first passed through a Kalman filter to extract smooth signals for the estimation of angular acceleration as defined by (2) and for input into the theta estimation equations of (3) or (10). The state transformation matrix (F) and visibility matrix (H) for this filter is simply the identity matrix. The sensor noise matrix $(\mathbf{F})$ contains the measured variance of the raw accelerometer sensor signal along the diagonal. Both the control transformation matrix $(\mathbf{G})$ and the control input $(\vec{u})$ are set to zero as this is a reactive measurement; ie. it has no knowledge of the system control inputs. The process noise matrix $(\mathbf{V})$ cannot be measured and is tuned to provide the best results during testing. As V behaves much like the time constant in traditional lowpass filters, the tuning involves a balance between phase lag and smooth signals. The filter is initialized with the first measurement and associated measurement noise.

The smoothed accelerometer measurements are then converted to angular acceleration according to (2) and combined with angular velocity, measured by the rate gyro, and the inclination estimate, calculated from (3) or (10), in a second Kalman Filter. The parameters for this filter are described in (13). This filter relies on a unbiased estimate of angle which can be obtained from either (3) or (10) depending on if the system can be treated as static or dynamic. To decide between (3) and (10) a measure of the magnitude of dynamic motion is required. Combining the magnitudes of the centripetal and angular acceleration terms in (1) leads to defining the dynamic magnitude by (14). This definition is arbitrary as it only needs to distinguish between variances for dynamic estimates and static estimates. The definition defined in (14) was experimentally verified to be adequate. If $d$ is is greater than a threshold value ( $d_{\text {Threshold }}$ ) the system is considered dynamic and (10) is used if it is below (3) is used. The calculation of $d_{\text {Threshold }}$ is discussed in further detail below. The value of $\hat{\theta}$ to be used in (10) is calculated by simply multiplying the previous state by the state transition matrix $\mathbf{F}$.

$$
\begin{gathered}
\mathbf{H}=\left[\begin{array}{lll}
1 & 0 & 0 \\
0 & 1 & 0 \\
0 & 0 & 1
\end{array}\right], \mathbf{F}=\left[\begin{array}{ccc}
1 & T & \frac{T^{2}}{2} \\
0 & 1 & T \\
0 & 0 & 1
\end{array}\right], \\
\mathbf{V}=\left[\begin{array}{ccc}
1 \times 10^{-6} & 0 & 0 \\
0 & \frac{1}{10} \sigma_{\dot{\theta}}^{2} & 0 \\
0 & 0 & \frac{1}{10} \sigma_{\ddot{\theta}}^{2}
\end{array}\right], \\
\mathbf{W}=\left[\begin{array}{ccc}
\sigma_{\theta}^{2} & 0 & 0 \\
0 & \sigma_{\dot{\theta}}^{2} & 0 \\
0 & 0 & \sigma_{\ddot{\theta}}^{2}
\end{array}\right], \mathbf{G}=\mathbf{0} \\
d=\sqrt{\ddot{\theta}^{2}+\dot{\theta}^{4}}
\end{gathered}
$$

\section{B. Inclination Measurement Noise Characterization}

The performance of the Kalman filter relies on correct characterization of the noise in the system. For the rate gyros and accelerometers this noise is easily measured. The error propagation through (10) and (3) is more easily simulated than calculated or measured as it is a function of the true inclination angle, the accelerometer measurements, and the accelerometer measurement variances. To estimate the variance under different conditions, the kinematics described in (1) are calculated with random inputs of angle, angular acceleration, and angular velocity generating a random list of possible states for the accelerometers. These states are then overlayed with a normally distributed random noise that has a variance equal to the variance measured in the smoothed acceleration data. This, now noisy, list of simulated accelerometer measurements is passed through (10) and (3) to calculate the estimated angle given the noise and dynamic accelerations. This angle is compared to the correct angle used in (1) to generate the accelerometer measurements and an error is calculated. This error, and its associated dynamic magnitude $d$ calculated according to (14), is sorted for statistical processing. A plot of variance vs dynamic magnitude is shown in Fig. 2 for both $\theta_{\text {Static }}$ and $\theta_{\text {Dynamic }}$. As expected, the variance of $\theta_{\text {Static }}$ is smaller for less dynamic motions and the variance of $\theta_{\text {Dynamic }}$ is smaller for more dynamic motions. The dynamic magnitude that these two variances cross determines $d_{\text {Threshold }}$. The static variance increases with dynamic motions and a quadratic function fits the variance of the static solution below $\theta_{\text {Threshold }}$ well. This fit can be used to express the variance for the measurement noise matrix $(\mathbf{W})$ as a function of dynamic magnitude. The dynamic variance to be used in $\mathbf{W}$ is defined by the piece wise continuous function of estimate noise given in (15) where the choice of (10) or (3) to calculate angle switches at $d=d_{\text {Threshold. }}$. The constants $\mathrm{A}, \mathrm{B}$, and $\mathrm{C}$ in (15) are obtained by a least-squares fitting of a quadratic function to the static solution variance data for dynamic magnitudes up to and including the threshold value.

$$
\sigma_{\theta}^{2}=\left\{\begin{array}{cc}
A d^{2}+B d+C & \text { if } d<d_{\text {Threshold }} \\
\sigma_{\text {dynamic }}^{2} & \text { if } d \geq d_{\text {Threshold }}
\end{array}\right.
$$

\section{Testing}

The sensor fusion technique discussed above was tested on an IMU constructed with two ADXL-325 $\pm 3 \mathrm{~g}$ tri-axis accelerometers and one ADXRS610 $\pm 300 \mathrm{deg} / \mathrm{sec}$ rate gyro from Analog Devices ${ }^{1}$. The two accelerometers are mounted $120 \mathrm{~mm}$ apart with the rate gyro mounted in the middle. The device is shown in Fig. 3. The IMU is mounted to a Quanser ${ }^{2}$ SRV-02 rotational link servo and subjected to a number of trajectories to determine the characteristics of the IMU and filter methodology. A series of constant angular velocity inputs and sinusoidal inputs are given to the SRV-02 hardware under close loop control. The inclination

\footnotetext{
${ }^{1}$ www.analog.com

${ }^{2}$ www.quanser.com
} 

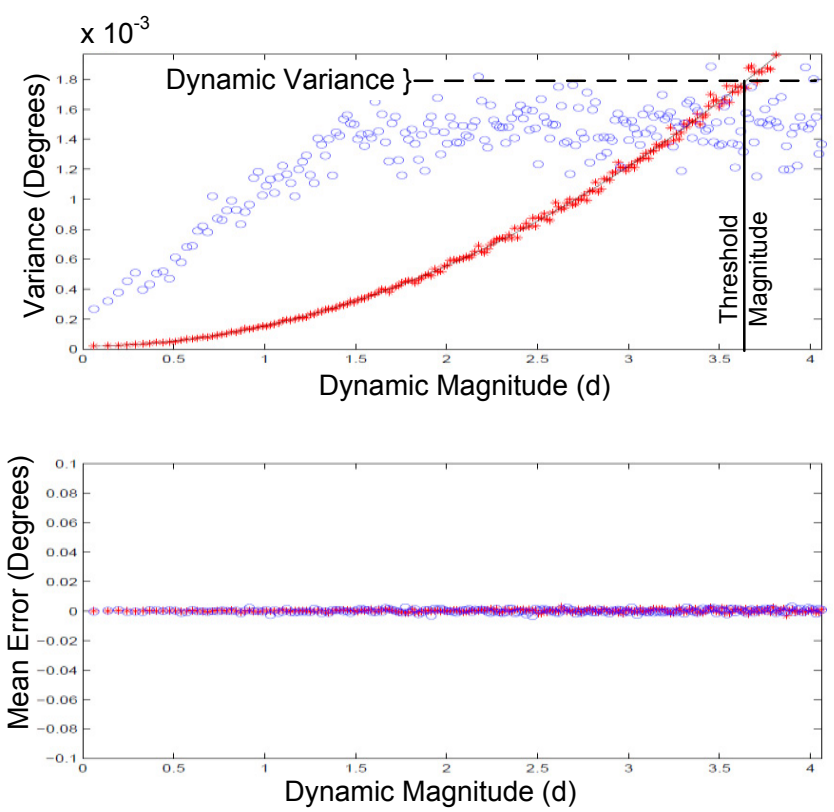

Fig. 2. The Variance (top) and Mean (bottom) of the Error in radians of $\theta_{\text {Static }}(*)$ and $\theta_{\text {Dynamic }}(\mathrm{o})$ as a function of dynamic magnitude. This simulation shows the optimal static vs dynamic solution crossover threshold $\left(d_{\text {Threshold }}\right)$ at a dynamic magnitude of 3.6.

estimate from the IMU and filter is compared to the encoder angle measurement from the SRV-02 to characterize the performance of the algorithm discussed above. All control and filtering is accomplished using the real time target feature of Matlab Simulink ${ }^{\circledR 3}$.

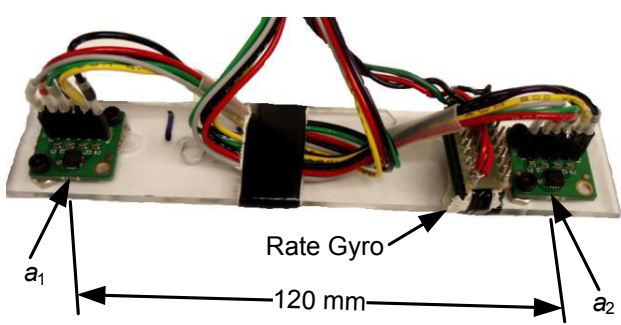

Fig. 3. The tested IMU comprised of two tri-axis accelerometers separated by $120 \mathrm{~mm}$ and one uni-axis rate gyro.

\section{A. Test Results}

The IMU is put through a series of constant angular velocity inputs with increasing rotational rate and sinusoidal inputs with increasing frequency. For each of the test cases the IMU is initially held stable for approximately one second to allow for a well characterized static measurement of the initial inclination angle. The responses calculated from the IMU are compared against the encoder readings available from the SRV-02. A plot of mean error and RMS error for both the constant velocity and sinusoidal inputs are shown as a function of input rate in Fig. 4 and Fig. 5, respectively.

\footnotetext{
${ }^{3}$ www.mathworks.com
}

In all of the testing, no indication of drift was detected. A comparison of the filter using the approach described in the paper and the filter using just the rate gyro to estimate inclination is shown in Fig 6 for a $1.0 \mathrm{~Hz}$ sinusoidal input; clearly, the use of $\theta_{\text {Dynamic }}$ prevented estimate drift in this case. With the exception of one outlier, the maximum error recorded in inclination estimate relative to the SRV-02's encoder is less than twenty degrees with the majority of the error less than five degrees.
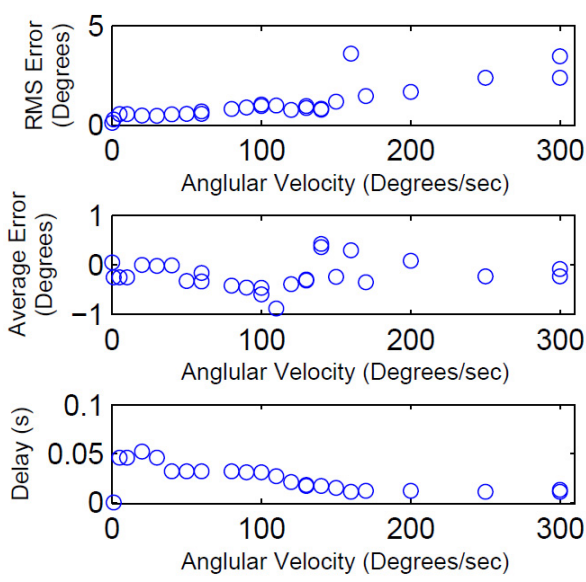

Fig. 4. Performance of the algorithm to different constant angular velocity inputs. Max RMS error is less than four degrees.
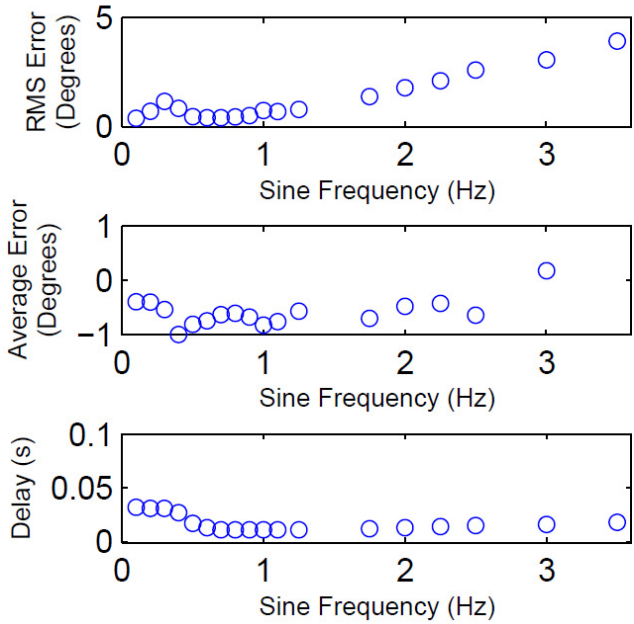

Fig. 5. Performance of the algorithm to different sinusoidal inputs. The maximum RMS error is less than four degrees.

\section{CONCLUSIONS AND FUTURE WORK}

The IMU and filtering method presented shows good performance to static, constant velocity, and sinusoidal inputs. There is no indication of drift recorded during any of the tests and the RMS error shows agreement in angle measurement with the encoder to less than four degrees during dynamic trajectories and less than one degree to a 


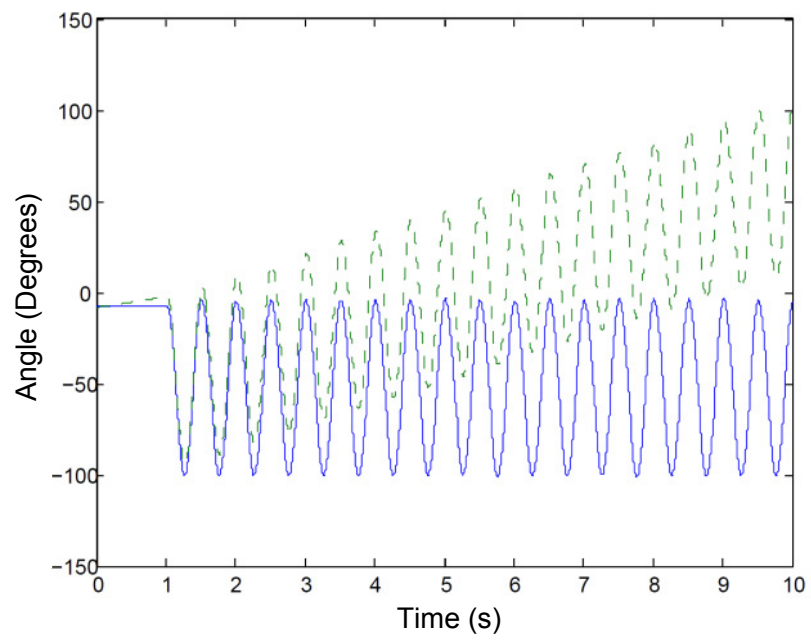

Fig. 6. A comparison between the algorithm implemented with the dynamic angle estimation (solid) and without (dashed). It is clear that the dynamic angle estimation removes drift from the system.

static position. Future work will consist of implementing this sensor system in a compact form and conducting testing in a gait laboratory to determine the performance of the system under actual sitting, walking, and running conditions.

\section{REFERENCES}

[1] K. Tong and M. Granat, "A practical gait analysis system using gyroscopes," Medical engineering \& physics, vol. 21, no. 2, pp. 87-94, 1999.

[2] R. Williamson and B. Andrews, "Detecting absolute human knee angle and angular velocity using accelerometers and rate gyroscopes," Medical and Biological Engineering and Computing, vol. 39, no. 3, pp. 294-302, 2001.

[3] Y. Yamamoto, K. Jikuya, T. Nakamura, T. Kusuhara, H. Michinishi, and T. Okamoto, "Precise measurement system for knee joint motions at pendulum test using accelerometer-detection of angular acceleration by two linear accelerometers-," in World Congress on Medical Physics and Biomedical Engineering, September 7-12, 2009, Munich, Germany. Springer, 2009, pp. 165-168.

[4] R. Hyde, L. Ketteringham, S. Neild, and R. Jones, "Estimation of upper-limb orientation based on accelerometer and gyroscope measurements," Biomedical Engineering, IEEE Transactions on, vol. 55, no. 2, pp. 746-754, 2008.

[5] R. Mayagoitia, A. Nene, and P. Veltink, "Accelerometer and rate gyroscope measurement of kinematics: an inexpensive alternative to optical motion analysis systems," Journal of Biomechanics, vol. 35, no. 4, pp. 537-542, 2002.

[6] K. O'Donovan, R. Kamnik, D. O'Keeffe, and G. Lyons, "An inertial and magnetic sensor based technique for joint angle measurement," Journal of biomechanics, vol. 40, no. 12, pp. 2604-2611, 2007.

[7] A. Sabatini, "Quaternion-based extended kalman filter for determining orientation by inertial and magnetic sensing," Biomedical Engineering, IEEE Transactions on, vol. 53, no. 7, pp. 1346-1356, 2006.

[8] Y. S. Suh, "Orientation estimation using a quaternion-based indirect kalman filter with adaptive estimation of external acceleration," Instrumentation and Measurement, IEEE Transactions on, vol. 59, no. 12, pp. $3296-3305$, December 2010 .
[9] A. Olivares, J. M. orriz, J. RamArez, and G. Olivares, "Accurate human limb angle measurement: Sensor fusion through kalman, least mean squares and recursive least-squares adaptive filtering," Measurement Science and Technology, vol. 22, no. 2, 2011. [Online]. Available: www.scopus.com

[10] H. Luinge and P. Veltink, "Measuring orientation of human body segments using miniature gyroscopes and accelerometers," Medical and Biological Engineering and Computing, vol. 43, no. 2, pp. 273 282, 2005.

[11] G. Cooper, I. Sheret, L. McMillian, K. Siliverdis, N. Sha, D. Hodgins, L. Kenney, and D. Howard, "Inertial sensor-based knee flexion/extension angle estimation," Journal of biomechanics, vol. 42, no. 16 , pp. 2678-2685, 2009.

[12] H. Dejnabadi, B. Jolles, E. Casanova, P. Fua, and K. Aminian, "Estimation and visualization of sagittal kinematics of lower limbs orientation using body-fixed sensors," Biomedical Engineering, IEEE Transactions on, vol. 53, no. 7, pp. 1385-1393, 2006.

[13] H. Dejnabadi, B. Jolles, and K. Aminian, "A new approach to accurate measurement of uniaxial joint angles based on a combination of accelerometers and gyroscopes," Biomedical Engineering, IEEE Transactions on, vol. 52, no. 8, pp. 1478-1484, 2005.

[14] P. Cheng and B. Oelmann, "Joint-angle measurement using accelerometers and gyroscopes - a survey," Instrumentation and Measurement, IEEE Transactions on, vol. 59, no. 2, pp. 404-414, 2010.

[15] J.-S. Hu and K.-C. Sun, "Human gait estimation using a reduced number of accelerometers," in SICE Annual Conference 2010, Proceedings of, Taipei, 2010, pp. 1905-1909.

[16] A. Tomaru, S. Kobashi, Y. Tsumori, S. Yoshiya, K. Kuramoto, S. Imawaki, and Y. Hata, "A 3-dof knee joint angle measurement system with inertial and magnetic sensors," in Systems Man and Cybernetics (SMC), 2010 IEEE International Conference on, October 2010, pp. $1261-1266$.

[17] L. MacFadden, "Musculoskeletal modeling for intrafascicular multielectrode stimulation of the feline hindlimb," Ph.D. dissertation, University of Utah, 2010.

[18] R. E. Kalman, "A new approach to linear filtering and prediction problems," Journal Of Basic Engineering, vol. 82, no. Series D, pp. $35-45,1960$.

[19] H. Choset, K. M. Lynch, S. Hutchinson, G. Kantor, W. Burgard, L. E. Kavraki, and S. Thrun, Principles of Robot Motion: Theory, Algorithms, and Implementations, R. C. Arkin, Ed. MIT Press, 2005. 